\title{
GLAD!
}

Revue sur le langage, le genre, les sexualités

$10 \mid 2021$

Varia

\section{Pédagogies féministes et didactique des langues- cultures : des liens à construire}

Entretien avec les membres de GIFLEX pour GLAD!

Creating Links between Feminist Pedagogies and Language and Culture

Teaching. An Interview with GIFLEX Members for GLAD!

Sophie Bailly, Véronique Lemoine-Bresson et Carine Martin

\section{OpenEdition}

Journals

Édition électronique

URL : https://journals.openedition.org/glad/2694

DOI : 10.4000/glad.2694

ISSN : 2551-0819

Éditeur

Association GSL

\section{Référence électronique}

Sophie Bailly, Véronique Lemoine-Bresson et Carine Martin, « Pédagogies féministes et didactique des langues-cultures : des liens à construire », GLAD! [En ligne], 10 | 2021, mis en ligne le 15 juillet 2021, consulté le 26 juillet 2021. URL : http://journals.openedition.org/glad/2694 ; DOI : https://doi.org/ 10.4000/glad.2694

Ce document a été généré automatiquement le 26 juillet 2021.

\section{c) (1) $\odot$}

La revue GLAD! est mise à disposition selon les termes de la Licence Creative Commons Attribution -

Pas d'Utilisation Commerciale - Pas de Modification 4.0 International. 


\title{
Pédagogies féministes et didactique des langues-cultures : des liens à construire
}

\author{
Entretien avec les membres de GIFLEX pour GLAD! \\ Creating Links between Feminist Pedagogies and Language and Culture \\ Teaching. An Interview with GIFLEX Members for GLAD!
}

Sophie Bailly, Véronique Lemoine-Bresson et Carine Martin

Julie Abbou. Pour commencer, pouvez-vous me parler de votre groupe Giflex?

Sophie Bailly. Giflex, c'est un nom de code pour désigner une recherche interdisciplinaire, voire indisciplinaire. Le projet de Giflex est de chercher à construire des liens entre pédagogies féministes et didactique des langues-cultures, dans le contexte de la formation à l'université. Nous sommes trois enseignantes-chercheuses de l'université de Lorraine, impliquées dans des contextes de formation différents, l'Institut National Supérieur du Professorat et de l'Éducation (INSPE), l'UFR LANSAD ${ }^{1}$ et le département Sciences du langage. Nous sommes membres d'une même équipe de recherche au sein du laboratoire ATILF. Pour chacune d'entre nous, les questions liées au genre et au féminisme font partie de nos cours, soit parce qu'elles sont explicitement enseignées, ou parce qu'elles émergent à partir des préoccupations des publics formés.

J.A. Dans la mesure où vous travaillez en recherche sur vos terrains de formation, vous formez un groupe de recherche appliquée?

Véronique Lemoine-Bresson. Comme en effet, nous nous centrons sur nos pratiques d'enseignement et celles d'apprentissage à l'université, il est difficile de ne pas avoir une posture impliquée. Le terme appliquée fonctionne mal par contre. Il suppose qu'il y aurait une subordination évidente entre théorie et pratique, c'est la dérive applicationniste. On travaille plutôt dans une démarche de recherche qui part de nos terrains, qui relève les problèmes rencontrés par nos publics, et par nous-mêmes. On peut dire que notre groupe de recherche travaille dans une visée de transformation, à 
double titre, par les pédagogies qu'on met en place dans nos cours, avec un retour réflexif qui mène à des transformations de postures pour les étudiantes et les étudiants, et à des transformations de pratiques pour nous.

J.A. Quelle différence faites-vous entre pédagogie et didactique ? Les féministes parlent toujours de pédagogie ; les linguistes parlent toujours de didactique.

S.B. C'est une question intéressante. Donc, didactique, on regarde plutôt la dimension des dispositifs, des organisations et des contenus d'enseignement et d'apprentissage. Si on prend mon exemple et celui de Carine, on a toutes les deux un cours qui porte sur le genre. On se centre donc sur tout ce qui est objets d'enseignement et d'apprentissage, implicites ou explicites en lien avec ce domaine. On cherche à inscrire nos dispositifs d'enseignement dans des formes qui soient en rupture avec ce qu'on pourrait appeler des formes pédagogiques inscrites dans le système patriarcal, au sens de système de domination qui donne les pleins pouvoirs à l'enseignante dans un rapport asymétrique. Il s'agit plus communément des pédagogies dites classiques, c'est-à-dire où un enseignement magistral domine, étant le même pour toutes les étudiantes et tous les étudiants, l'enseignante "tout sachant» étant dans une posture transmissive. Pour la pédagogie, on serait peut-être plus sur des questions de relation enseignant·e-enseigné'e ou professeur·e-élève, enseignant·e-apprenant·e, et du rôle de chacunee, et également sur la question de la passation de consigne, peutêtre celle des questions d'évaluations dont les fonctionnements varient selon les pédagogies (classiques ou différentes).

V.L.B. Oui, Julie, tu poses une question très intéressante et complexe. Ta question est d'autant plus complexe que les termes mêmes didactique(s) et pédagogie(s) peuvent ne pas recouvrir la même importance ou la même signification selon les espaces géographiques qui les emploient. Ici, nous les exprimons en français, en France, et nous tentons d'en donner deux définitions distinctes. On peut penser que lorsqu'ils sont exprimés dans deux langues différentes, leur compréhension et leur définition s'inscrivent dans des cadres de pensée propres aux deux espaces géographiques. Ces termes sont marqués d'une certaine tradition, d'une histoire et du sens donné à l'éducation, mais aussi le mot pédagogie peut être relié à des idéologies et à des politiques éducatives, selon les époques et les contextes. Quant au mot didactique, je me demande s'il existe dans toutes les langues. Même en France, je ne suis pas certaine qu'on s'entende sur sa définition. Si on parle de notre groupe, Sophie et Carine ont des contenus de cours à enseigner qui ont leurs propres caractéristiques et sont inscrits dans des parcours universitaires spécifiques. Ces cours peuvent être optionnels ou obligatoires, s'adresser à des étudiants d'âge différents avec des savoir déjà-là plus ou moins développés, et ces critères peuvent jouer sur les pratiques de classe. Les contenus enseignés des cours de Carine et Sophie touchent explicitement au genre tandis qu'en ce qui me concerne, j'ai des contenus d'enseignement qui touchent plutôt à la question de l'interculturalité. Et la question du genre, en fait, elle émerge dans mon cours par le biais des étudiantes, en situation qui profitent de la définition «large» que je construis avec eux de l'interculturalité. Elles et ils y problématisent des expériences individuelles et collectives qui articulent la question "des cultures» et "du genre ", par la mise au jour de rapports de domination, de discriminations, de racisme, de sexisme. Le travail sur ces contenus va faire appel à des savoirs disciplinaires, aux notions de connaissances déclaratives, procédurales, et de compétences qui se construisent dans les expériences à travers des tâches, des 
activités. Et après, tout ce qui touche à la pédagogie c'est effectivement quand on met à l'épreuve de nos étudiantes ces contenus inscrits dans une institution (master à l'INSPE; licence à l'UL), dans un contexte particulier, contenus qui vont devenir des contenus à enseigner, puis enseignés et peut-être appris. Qu'est-ce qui va servir d'appui à la pratique concrète en cours et pour quelles finalités? Quelles conceptions et représentations, ou encore quels savoirs d'action pédagogique validés par la recherche vont servir de base à nos choix pédagogiques pour nos pratiques? Qu'estce qu'on met en place comme dispositifs au cœur d'un curriculum spécifique, qu'estce qu'on active comme savoirs, savoirs d'expériences, savoirs professionnels, savoirs déjà-là des étudiantes et leurs besoins pour proposer concrètement un parcours d'apprentissage pour nos étudiantes? La pédagogie a ainsi deux dimensions fondamentales, l'enseignement des contenus et la gestion de notre cohorte d'étudiant·es pour qu'elles et ils apprennent. La pédagogie et de nombreux domaines scientifiques permettent d'éclairer les différentes didactiques. Concernant la pédagogie féministe ${ }^{2}$, on voit l'influence des études de genre, mais aussi des savoirs des enseignantes dans les années 1960 au sujet de la réalité sociale des femmes et de leur motivation à changer les choses, notamment en éducation, dans les classes. Dans nos propres cours d'ailleurs, on peut dire que nos pratiques renvoient parfois à des événements « réels » de l'espace social qui servent alors de référence pour concevoir les activités qu'on propose. Il me semble que la pédagogie féministe, au-delà du questionnement de la réalité sociale des femmes, tend à impulser des actions pour une transformation des rapports de domination liés au genre. Dans Giflex, nous nous intéressons à nos manières de construire nos objets d'enseignement, pour une meilleure compréhension de nos fonctionnements pédagogiques. Il s'agit d'essayer d'activer une pédagogie différente de la pédagogie classique, et de s'inscrire dans une pédagogie féministe, dont nous devons identifier les principes fondamentaux et les principes d'action à partir de nos pratiques et de la littérature.

J.A. C'est quoi pour vous la pédagogie féministe? Qu'est-ce que ça amène par rapport à la pédagogie critique, par exemple?

Carine Martin. Je pense qu'il faut revenir sur l'histoire de cette pédagogie pour en saisir les enjeux. La pédagogie féministe, ou plutôt les pédagogies féministes, sont apparues dans les années 1980 dans les pays anglophones, en particulier les ÉtatsUnis et le Canada. L'élément-clé dans le développement de ces pédagogies, c'est l'essor des départements de Women's Studies qui mettent au point des programmes spécifiques ainsi que leurs propres théories et méthodologies. On voit aussi l'émergence, à ce moment-là, de sociétés savantes et de publications spécifiques comme Feminist Teacher. Le terme lui-même apparaît au début des années $1980^{3}$. Le lien entre les pédagogies féministes et les pédagogies critiques (ou d'émancipation) a donné lieu à de nombreux débats. Est-ce qu'un rapport de filiation existe ou est-ce qu'il faut les concevoir comme deux pédagogies séparées, mais complémentaires ? En tout état de cause, il y a des liens multiples et des influences mutuelles. Par exemple, les deux approches ont en commun un projet social d'émancipation à travers une prise de conscience critique. Mais il y a également des différences, certaines théoriciennes de la pédagogie féministe ont notamment souligné la dimension paternaliste des débuts de la pédagogie critique. Les recherches en pédagogies féministes se font sous l'angle du genre, ce qui n'est pas le cas des pédagogies critiques. Il y a aussi une remise en question du rapport enseignant-enseigné et des rapports de pouvoir qui peuvent se nouer au sein même de la classe, et qui ne sont 
pas souvent abordés par les pédagogies critiques.

Depuis les années 1990, les pédagogies féministes se sont enrichies des apports du féminisme intersectionnel et ont ajouté d'autres types de discrimination (classe origine ethnique - identité sexuelle) à leur approche. En fait, il faut plutôt voir les pédagogies féministes comme une constellation. Par exemple, à l'intérieur des pédagogies féministes, il y a la Black Feminist Pedagogy. Et cette constellation peut être plus ou moins reliée à d'autres types de pédagogies. Ainsi, il y a une interconnexion avec les pédagogies décoloniales, qui s'intéressent beaucoup au système de genre comme instrument d'assujettissement des indigènes par les colons. bell hooks est un exemple célèbre de cette approche.

S.B. Je pense que qualifier une pédagogie de féministe met un coup de projecteur sur le fait que nos choix pédagogiques ne sont pas neutres politiquement et éthiquement, si l'on comprend pédagogie féministe comme la mise en œuvre dans une classe de pratiques d'enseignement qui promeuvent l'égalité de traitement entre les filles et les garçons, par exemple, ou qui permettent de prendre conscience des biais de genre dans la situation d'enseignement. Cette pédagogie féministe, comme dit Carine, c'est tout aussi bien, ou tout à la fois, une pédagogie inclusive, critique, émancipatrice, coopérative, transformatrice, etc. Mais ce qui est intéressant aussi c'est que le mot féministe associé au mot pédagogie m'invite à imaginer les notions de pédagogie non féministe, sexiste, antiféministe, ou carrément pédagogie patriarcale, qui seraient donc des pédagogies dont les modalités d'action seraient en tous points contraires à celles des pédagogies féministes. Au lieu d'émanciper les apprenant-es-étudiant·es, ces pédagogies les maintiendraient dans un rapport de domination, de soumission (au prof, au système, aux idéologies liées notamment aux marchés, etc.). La notion de pédagogie féministe nous invite à réfléchir à ce qui, dans les modes de transmission universitaires classiques - dits cours magistral, TD et TP - contribue à faire de l'université plus un lieu de compétition, de sélection et de reproduction des structures du pouvoir, qu'un lieu où chacune et chacun peut réussir, et s'épanouir en tant qu'individu et en tant que membre d'une société.

V.L.B. En fait, si je dois répondre pour mon cours sur l'interculturalité à l'INSPE, donc pour la formation à l'enseignement scolaire, cette notion de pédagogie féministe m'a permis d'être plus attentive à faire émerger les questions d'ethnie, race, classe sociale, genre, etc. Avant, c'était une catégorie parmi d'autres, en quelque sorte. Et du coup, ça me permet maintenant d'en prendre plus conscience. Tous les ans, cette question ressort, ce n'est pas quelque chose qui apparaît de temps en temps, mais dès que les étudiants ont l'opportunité de proposer des séances pédagogiques pour la classe, cette question du genre émerge systématiquement. Avoir en tête le mot féministe me permet de m'y intéresser un peu plus, d'être plus attentive à ce rapport de pouvoir genré auquel je faisais attention, certes, mais pas plus que ça avant la création de Giflex.

S.B. Oui pour moi c'est pareil, c'est plus comme une espèce de petit signal d'alarme. Il me semble que la notion de pédagogie féministe amène à interroger les modèles de rapport prof-élève ainsi que les espaces et les formats de cours et ce qu'ils doivent peut-être au système hétéro-patriarcal. Par exemple, dans un cours d'anglais scientifique que je donne en master de didactique des langues, sans contenus de genre, j'apprends à mes étudiantes à s'émanciper en tant qu'apprenantes et à découvrir leur propre façon d'apprendre : cet objectif est commun aux paradigmes 
des pédagogies autonomisantes, que je pratique pour ce cours, et aux pédagogies féministes. Par contre, je donne un cours de genre et langage en première année de licence, dans un amphi pour plus de 150 étudiants, et là c'est très difficile - mais pas impossible - de mettre en place des modalités autres que le cours magistral traditionnel où le savoir descend du maître sachant vers l'élève ignorant ${ }^{4}$, en établissant des rapports de pouvoirs moins inégalitaires. Il est clair qu'il peut y avoir des abus de pouvoir dans certaines manières d'enseigner et d'encadrer. D'ailleurs, on a eu un cas très dramatique qui s'est déroulé à l'UL l'été dernier, le suicide d'une doctorante accompagné de l'incrimination de son encadrant de thèse. Cette tragédie met sur la sellette le système mandarinal auquel peut aboutir cette conception patriarcale des rapports sociaux y compris au sein de l'institution universitaire. Donc pour répondre à ta question oui, le mot féministe fonctionne pour nous comme une espèce de code pour faire ce pas de côté et regarder les choses avec un autre regard et développer une réflexivité pour transformer son agir professoral.

J.A. Est-ce que la pédagogie féministe peut aider à gérer les résistances des étudiants quand on enseigne les thématiques liées au genre et aux sexualités? D'ailleurs, j'ai remarqué que c'est principalement des garçons qui résistent.

C.M. Oui tout à fait, la pédagogie féministe nous aide à faire deux choses : comprendre d'où vient cette résistance masculine et développer des stratégies pour la transformer. Les étudiants peuvent se sentir menacés parce que le contenu du cours vient remettre en question les privilèges de la masculinité auxquels ils pensaient naïvement pouvoir accéder et pour lesquels ils ont déjà fait beaucoup de sacrifices personnels ${ }^{5}$. Les hommes sont donc à la fois opprimés et oppresseurs et c'est en faisant découvrir cette contradiction à l'intérieur même de la masculinité qu'on peut aider les étudiants à se libérer. C'est important de reconnaître que l'étudiant est aussi victime du système. Le but d'une pédagogie féministe face à la résistance des étudiants est de les rendre conscients de leur expérience inconsciente et contradictoire du pouvoir et d'une façon qui laisse plus de place à l'intellect qu'aux émotions. Concrètement, cela peut commencer notamment par la présentation de la théorie, avant de passer à des études de cas, pour éviter qu'ils ne se sentent personnellement menacés.

S.B. Mais il peut arriver, surtout avec un public qui ne serait pas déjà en partie convaincu, que la théorie soit perçue comme de l'idéologie voire du prosélytisme militant. C'est ce que m'a fait remarquer un étudiant lors de l'évaluation finale par les étudiants de mon cours Genre et Langage. Il reproché au cours de véhiculer, je le cite: "des pensées de gauche basées sur le progressisme» pour "partager des idéologies $"$.

V.L.B. Le groupe peut jouer un rôle crucial sur un changement d'attitudes, ou une prise de conscience. J'ai pu observer dans mes cours que cette prise de conscience des places que les hommes peuvent prendre ou auxquelles ils peuvent être assignés dans la société n'est pas une évidence. Lors d'un cours, au moment d'interroger la notion de "culture du viol ", un étudiant, de façon très maladroite, voulait essayer d'apporter des éléments de justification de certaines agressions d'hommes envers les femmes, se posant comme "quelqu'un d'ouvert » et de "nuancé ». C'était un peu comme s'il y avait les "vrais viols » et ceux qui en avaient l'apparence, mais pas la consistance. Plus il avançait dans son raisonnement, moins il semblait conscient du fait qu'il minimisait le viol face à un groupe constitué à $98 \%$ de jeunes femmes... qui 
n'ont pas tardé à réagir vertement. Ce sont essentiellement ces réactions vives et massives suivies d'échanges, et non la parole de l'enseignante qui ont fait poids, l'amenant petit à petit (mais vraiment très lentement) à se rendre compte d'une part de son discours emprunt de mythes et de stéréotypes, et d'autre part des effets de ses paroles.

C.M. Je fais un cours de L3 pour les étudiantes d'études culturelles intitulé « Gender and Contemporary Culture " et j'ai la chance d'avoir un public averti qui choisit de suivre cet enseignement, donc je n'ai encore jamais rencontré ce genre de problème. Ceci dit, j'ai pris les devants et adapté la progression du cours de manière à éviter ce genre de réaction. Ma méthode inclut l'étude de la théorie en cours avec une réflexion personnelle à faire à la maison, sous forme d'écrit sur un carnet de bord. Donc la conscientisation vient assez vite. Je commence par des thématiques générales qui touchent tout le monde : par exemple, nous étudions un texte sur la socialisation au genre et je demande aux étudiant-es de réfléchir à la façon dont elles-mêmes et eux-mêmes ont été socialisées au genre. Cela amène déjà une certaine prise de recul et certain.es évoquent les différences entre les filles et les garçons au sein de leur famille. Ou encore, je me souviens d'un étudiant américain qui m'a parlé des mythes qui existaient dans sa famille autour de la masculinité comme le fait de "réussir " notamment par l'accumulation d'argent. Un peu plus loin dans le semestre, nous avons une à deux séances sur les masculinités, dont une qui met particulièrement le doigt sur les souffrances que peuvent engendrer ces idéaux de masculinité pour les garçons, à travers un documentaire. Je leur demande de faire le lien avec leur expérience personnelle dans leur cercle amical et familial. C'est une manière pour moi d'aborder cette contradiction à l'intérieur de la masculinité. C'est seulement ensuite qu'on aborde les séances sur la communauté LGBTQI et le féminisme, donc à un moment où les hommes du cours ont déjà eu l'occasion de réinterpréter leur expérience en tant qu'oppresseur/opprimé. Je pense sincèrement que si on veut éviter ou plutôt surmonter les résistances des étudiants, on ne peut pas se passer de séances sur la masculinité. Après tout, si les objectifs des pédagogies féministes sont inclusifs et émancipatoires, on ne peut pas laisser les hommes de côté.

V.L.B. La question des résistances et des contradictions me rappelle mon vécu alors que j'étais enseignante en REP dans les années 1990-2000, en banlieue lilloise. De manière empirique, j'ai pu remarquer que les questions sur la masculinité étaient aux prises avec la question du conflit de loyauté. Certains de mes élèves garçons se refusaient de bien travailler en classe et de réussir à l'école pour ne pas trahir leur famille ou leurs relations amicales «dans le quartier». Pour des raisons semblables, d'autres, voire les mêmes, étaient très mal à l'aise avec la question de l'égalité fillesgarçons, pour reprendre les termes de l'éducation nationale. Il y avait un décalage entre les valeurs que l'école essayait de transmettre et celles de la famille et du " quartier ", et notamment celles venant des convictions religieuses. Donc, le facteur de la loyauté au milieu et à ses valeurs peut également être une cause de résistance masculine au changement.

J.A. Du point de vue de la recherche, quels sont vos projets ?

S.B. Nous avons plusieurs projets en cours en ce moment. Tout d'abord un projet autour du cours de Gender Studies en anglais pour non-spécialistes de Carine dont nous étudions l'organisation et la mise en œuvre à partir de questions comme : dans quelles mesures les modalités pédagogiques du cours s'alignent-elles avec le projet 
émancipatoire et transformatif des pédagogies féministes et donc le soutiennent ? Quels sont les effets du cours (des contenus, des consignes, des modalités de transmission et de médiation, de l'organisation spatiale et temporelle, etc.) sur d'une part le rapport au genre et d'autre part sur l'acquisition de compétences langagières en anglais? Le cours étant basé sur l'utilisation d'un carnet de bord individuel, nous allons regarder aussi en quels termes les étudiants parlent de leur propre identité genrée. Quelle narration font-elles et font-ils de leur trajectoire par rapport à leur genre ? Comment est-ce que les étudiant-es comptent utiliser les outils théoriques et pratiques acquis pendant le cours dans leurs débats à l'extérieur de l'université ? Estce que cela les transforme, non seulement dans le cadre d'un cours à l'université, mais également dans les autres dimensions de leur vie sociale, dans leurs autres rôles sociaux en dehors de l'université?

Et nous sommes aussi sur le terrain de l'école grâce à une étudiante de Véronique qui participe à nos travaux.

V.L.B. C'est une étudiante de M2 MEEF, une future professeure des écoles qui travaille sur la question de l'injonction à l'égalité filles-garçons dans les programmes officiels de l'éducation nationale, en maternelle notamment. Pendant ses stages elle a remarqué la frilosité des enseignantes à sortir de la binarité de genre et à sortir d'activités pour dire vite, très prêtes à penser, très très convenues, qui ne bousculent pas trop les choses. Son idée a été, dès le master 1 , de proposer aux élèves de réfléchir au-delà d'une conception binaire, d'autant plus que les élèves ont dans leur besace, dans leurs connaissances et dans leurs expériences, des éléments qui sortent de la binarité. Ils connaissent, par exemple, l'artiste non binaire Bilal Hassani qui a représenté la France à l'Eurovision en 2019, et en discutent..., essentiellement dans la cour de récréation! Et du coup elle a proposé des ressources pédagogiques d'appui pour faire des séquences qui étaient un petit peu à la marge du binaire et ça a été très mal reçu dans les écoles. Elle s'est aperçue qu'il y avait peut-être une peur derrière ces réactions, plus que de l'immobilisme. Donc cette année, elle travaille sur les peurs des enseignantes vis-à-vis des ressources qui seraient fantasmées par les enseignant-es comme susceptibles de promouvoir la non-binarité du genre.

Mot de la fin. Nous remercions chaleureusement Julie Abbou pour son temps, son intérêt et ses questions pointues qui nous ont permis d'avancer dans notre réflexion. Nous sommes convaincues qu'une approche critique féministe de nos objets de recherche habituels en didactique des langues-cultures, dans la lignée des travaux pionniers en France, notamment ceux de Mireille Baurens, Véronique Perry et Nicole Décuré, peut contribuer à produire de nouveaux modèles pour repenser une université plus juste et plus égalitaire. Nous espérons pouvoir bientôt organiser une rencontre scientifique pour continuer d'échanger et débattre de ces recherches et pour les faire connaître, et nous invitons dès à présent toute personne intéressée à nous contacter. 


\section{NOTES}

1. Lansad = Langues pour spécialistes d'autres disciplines

2. Solar, C. 1992. "Dentelle de pédagogies féministes", Canadian Journal of Education/Revue canadienne de l'éducation, 17(3), 264-285.

3. Certaines chercheuses attribuent l'origine du terme à Berenice Fisher dans Fisher, B. 2018. «What is feminist pedagogy?» Nouvelles Questions Feministes, Vol. 37(2), 64-75.

4. Formule faisant écho à celle de Jacques Rancière dans son ouvrage Le maître ignorant (1987).

5. Voir Orr, D. J. 1993. «Toward a Critical Rethinking of Feminist Pedagogical Praxis and Resistant Male Students ", Canadian Journal of Education / Revue Canadienne de l'éducation, 18(3), 239-54.

\section{RÉSUMÉS}

Cet entretien avec les membres du groupe de recherche Giflex, né en 2020 à l'université de Lorraine au sein du laboratoire ATILF, porte sur les questionnements de trois enseignanteschercheuses en Didactique des langues-cultures aux prises avec les notions de genre et de féminisme sur leurs terrains de formation et au sein de leurs enseignements. Celles-ci abordent des questions théoriques et des cas pratiques et présentent les projets de recherche en cours.

This interview with the members of the Giflex research group, created in 2020 at the University of Lorraine within the laboratory CNRS ATILF, focuses on the reflections of three teacherresearchers in Didactics of Language and Culture who are grappling with the notions of gender and feminism in their teaching. They address theoretical questions, as well as practical examples and present current research projects.

\section{INDEX}

Thèmes : Explorations

Mots-clés : pédagogies féministes, didactique des langues-culture, enseignement supérieur

Keywords : feminist pedagogies, didactics of language and culture, higher education

\section{AUTEURS}

\section{SOPHIE BAILLY}

Sophie Bailly est professeuse d'université en sociolinguistique et didactique des langues. Elle enseigne principalement en master didactique des langues et en licence sciences du langage à l'université de Lorraine, et elle encadre des thèses. Ses recherches portent principalement, en didactique des langues-cultures, sur la question de l'autonomisation de l'apprentissage chez des apprenants de langues étudiants et adultes; et en sociolinguistique, sur le rapport genre et 
langage, domaine de sa thèse doctorale à Paris Descartes. Elle est à l'origine du projet Giflex, un groupe de réflexion et de recherche indisciplinaire autour des liens à construire entre la didactique des langues-cultures et les études de genre. Elle est l'autrice de l'ouvrage Les hommes, les femmes et la communnication. Mais que vient faire le sexe dans la langue? Ed. L'Harmattan.

\section{VÉRONIQUE LEMOINE-BRESSON}

Véronique Lemoine-Bresson est maîtresse de conférences à l'université de Lorraine. Elle est membre du laboratoire ATILF (CNRS), dans l'équipe didactique des langues et sociolinguistique. Elle enseigne en Master MEEF à l'Institut National Supérieur du Professorat et de l'Éducation de Lorraine et à l'Inspé des Hauts-de-France et à l'Institut de Formation Pédagogique de Lille. Elle s'intéresse particulièrement aux problématiques liées à l'interculturel, dans une articulation entre la formation théorique et pratique. Elle a travaillé dans l'Éducation nationale, en tant qu'enseignante en primaire et comme conseillère pédagogique départementale en langues, dans l'Académie de Lille. Ses travaux récents sont publiés dans Psychology of Language Learning and Teaching, Recherches en Didactiques (RED) et dans la Revue Internationale de Pédagogie de l'Enseignement Supérieur (Ripes).

\section{CARINE MARTIN}

Carine Martin est maîtresse de conférences en anglais à l'UFR LANSAD de l'université de Lorraine. Elle est membre de l'équipe de recherche didactique des langues et sociolinguistique (Crapel) de l'ATILF, elle participe notamment à l'axe « Autonomie et apprentissage autodirigé ». Elle enseigne l'anglais de spécialité au niveau master, elle fait également un cours d'initiation aux études sur le genre au niveau L3 sous un format Emile (Enseignement d'une matière intégrée à une langue étrangère). Elle est également conseillère en Centre de langue et responsable du projet eTandem pour l'UFR LANSAD. Ses recherches portent sur l'autonomisation de l'apprenant, et plus précisément sur l'accompagnement en ligne ainsi que l'accompagnement de l'apprenant dans le cadre du e-tandem et de l'entretien-conseil. Elle s'intéresse également à la transformation des pratiques pédagogiques via le numérique, et plus particulièrement à la pédagogie hybride. Après une thèse à la croisée de la civilisation britannique et des gender studies, elle cherche à présent à intégrer la dimension du genre dans ses recherches en didactique à travers le projet Giflex. 